\title{
TGFB1 polymorphisms are associated with risk of late normal tissue complications in the breast after radiotherapy for early breast cancer
}

\author{
Christian Nicolaj Andreassen ${ }^{\mathrm{a}, *}$, Jan Alsner ${ }^{\mathrm{a}}$, Jens Overgaard ${ }^{\mathrm{a}}$, Carsten Herskind $^{\mathrm{b}}$, \\ Jo Haviland ${ }^{c}$, Roger Owen ${ }^{d}$, Janis Homewood ${ }^{c}$, Judith Bliss ${ }^{c}$, John Yarnold ${ }^{\mathrm{e}}$
}

\author{
${ }^{a}$ Department of Experimental Clinical Oncology, Aarhus University Hospital, Denmark, 'bepartment of Radiation Oncology, \\ University Hospital Mannheim, Germany, ' Clinical Trials \& Statistics Unit, Section of Clinical Trials, Institute of Cancer Research, Sutton, UK, \\ ${ }^{\mathrm{d}}$ Gloucestershire Oncology Centre, Cheltenham, UK, ${ }^{\mathrm{e}}$ Section of Radiotherapy, Institute of Cancer Research, Sutton, UK
}

\begin{abstract}
Recent studies suggest that normal tissue radiosensitivity is influenced by single nucleotide polymorphisms (SNPs) in certain genes. In order to seek a confirmation of these findings, this study investigated SNPs in genes TGFB1 (position - 509, codon 10 and codon 25), SOD2 (codon 16), XRCC1 (codon 399), XRCC3 (codon 241), APEX (codon 148) and ATM (codon 1853) in 26 breast cancer patients with marked changes in breast appearance after radiotherapy and 26 matched controls. Statistically significant associations were found between the TGFB1 codon 10 Pro allele $(P=0.005)$ as well as the TGFB1 position -509 T allele $(P=$ 0.018 ) and increased risk of altered breast appearance. No significant associations were found for the remaining SNPs.

(C) 2005 Elsevier Ireland Ltd. All rights reserved. Radiotherapy and Oncology 75 (2005) 18-21.
\end{abstract}

Keywords: Normal tissue complications; Radiotherapy; Single nucleotide polymorphisms; Transforming growth factor beta 1

The ability to predict the risk of radiation induced normal tissue complications prior to cancer treatment has been a long sought goal in radiobiology. Currently, increasing efforts are made to investigate whether clinical normal tissue radiosensitivity is influenced by genetic germline variation $[1,6]$. A recently published study, based on a cohort of 41 Danish breast cancer patients given post-mastectomy radiotherapy, has indicated a possible association between selected single nucleotide polymorphisms (SNPs) and the risk of subcutaneous fibrosis after radiotherapy [2]. According to this study, the fibrosis risk was dependent on the number of 'risk alleles' possessed at the polymorphic sites TGFB1 position -509 and codon 10, XRCC 3 codon 241 , XRCC 1 codon 399 and SOD2 codon 16. A borderline significant association was observed for the TGFB1 codon 25 SNP whereas no obvious correlation was found for the APEX codon 148 SNP. In addition, the same patient cohort has lately been subjected to an investigation in which the ATM gene was systematically screened for genetic variation [15]. This study provided indications that the ATM codon 1853 Asp/Asn (G 5557 A) SNP may also affect risk of radiation induced fibrosis. Only a very limited number of other studies have addressed the influence of these SNPs upon normal tissue radiosensitivity $[4,9,10,12,14]$. In 2003, Quarmby et al. established significant associations between the TGFB1 position -509 and codon 10 SNPs and risk of subcutaneous fibrosis after post-lumpectomy radiotherapy for breast cancer [14]. Angele et al. demonstrated an association between the ATM codon 1853 Asn/Asn genotype and enhanced risk of various adverse reactions after radiotherapy for breast cancer [4]. Within the same patient cohort indications were found that the XRCC1 codon 399 SNP in combination with the relatively uncommon codon 194 SNP may also affect normal tissue radiosensitivity [12]. In 1998, a relatively small study reported a moderate overrepresentation of the ATM codon 1853 Asn allele among prostate cancer patients with severe late effects [10]. In order to seek a confirmation of the results obtained in the Danish patient cohort, the present study applied the analysis of the same SNPs to an independent patient cohort of women given radiotherapy after breast preserving surgery for breast cancer [18]. Subsets of this cohort have earlier been used for studies addressing the influence of truncating ATM mutations and SOD2 SNPs on normal tissue radiosensitivity $[9,16]$. In both studies, no significant associations were found between the investigated genetic variations and risk of altered breast appearance after radiotherapy. However, since the original SOD2 study differed slightly from the present with regard to patient selection and statistical analysis, the SOD2 codon 16 SNP (also referred to as SOD2 Ala-9Val) was also included in the present investigation. Furthermore, a relatively rare polymorphism designated ATM codon 1853 Asp/Val 
(A 5558 T) exists adjacent to the ATM codon 1853 Asp/Asn (G 5557 A) SNP. Since this polymorphism may potentially alter the amino acid incorporated at codon 1853, it was assessed as well.

\section{Material and methods}

The patients included in this study were given radiotherapy after breast preserving surgery for early stage breast cancer as part of a large radiotherapy fractionation trial at the Royal Marsden Hospital, Sutton and the Gloucestershire Oncology Centre, Cheltenham, UK. Patient characteristics, radiotherapy regimens, definition and assessment of endpoints have been reported in detail [18] and are therefore only described briefly here. Between 1986 and 1994, 935 patients were enrolled in the trial. Of these patients, 855 had baseline post-operative photographs of the breasts taken under standard conditions, which were repeated annually for 5 years. Changes in breast appearance compared to the post-surgical baseline photograph were scored on a three-point graded scale based on change in breast size and/or shape. Assessments were performed by three independent observers blind to patient identity, randomised radiotherapy dose and year of follow-up. Cases were defined as individuals developing marked changes (grade 2) at any time between 1 and 5 years after radiotherapy, or moderate changes (grade 1) scored for at least three consecutive years. Control subjects were defined as women with no change (grade 0 ) at the same time as the case experienced a reaction. From 104 of these patients (39 cases and 65 controls), fibroblast culture has previously been established [13]. For the purpose of the present study, 26 case-control pairs with accessible biological material and a perfect match regarding breast anatomy and treatment characteristics (breast size, surgical deficit, radiotherapy dose schedule, radiotherapy boost dose, tangential field separation, central lung distance and systemic therapy) could be identified.
Seven pairs were given 50.0 Gy in 25 fractions, 13 pairs were given $42.9 \mathrm{~Gy}$ in 13 fractions whereas 6 pairs received $39.0 \mathrm{~Gy}$ in 13 fractions.

DNA was extracted from cultured fibroblasts using the $A B I$ Prism $^{\mathrm{TM}} 6100$ Nucleic Acid PrepStation and the provided reagents according to the manufactures protocols (Applied Biosystems, Foster City, USA). From three patients, fibroblast culture was no longer accessible and DNA was isolated from fresh frozen lymphocytes or subcutaneous biopsies. The TGFB1 position - 509 and codon 10 SNPs were assessed by TaqMan ${ }^{\circledR}$ based real time PCR on the ABI Prism ${ }^{\circledR} 7000$ Sequence Detection System (Applied Biosystems, Foster City, USA). Primers and probes are listed in Table 1. For the remaining SNPs relevant DNA fragments were amplified by 35 rounds of thermal cycling $\left(95^{\circ} \mathrm{C}\right.$ for $30 \mathrm{~s}, 60^{\circ} \mathrm{C}$ for $30 \mathrm{~s}$ and $72{ }^{\circ} \mathrm{C}$ for $30 \mathrm{~s}$ ) using Amplitaq Gold polymerase (Applied Biosystems, Foster City, USA) and standard reaction conditions. PCR products were purified using a $4 \%$ agarose gel and the QIA-quick Gel Extraction Kit (Qiagen, Germany). The SNP genotypes were assessed by 'single nucleotide primer extension' using the $\mathrm{ABI}$ Prism ${ }^{\circledR} \mathrm{SNaPshot}^{\mathrm{TM}}$ Multiplex kit on an $A B I 310$ genetic analyzer (Applied Biosystems, Foster City, USA). PCR primers and extension primers are listed in Table 1. The analysis was conducted blind to patient identity, treatment characteristics, case-control status, etc. Redundant tests were carried out to assure assay reproducibility. For each SNP, a putative risk allele was defined according to the results obtained in the SNP studies previously conducted in the 41 Danish breast cancer patients $[2,15]$. With respect to the APEX codon 148 SNP, the minority allele (Glu) was arbitrarily chosen as putative risk allele. At each polymorphic site, the number of putative risk alleles could be 0,1 or 2 . The matched pairs were analysed as to whether the index case had a higher, lower or equivalent number of risk alleles compared to the control. A Wilcoxon signed ranks test for paired data was used to evaluate whether the distribution of number of risk alleles were

Table 1

Overview of assessed SNPs, primers and probes

\begin{tabular}{|c|c|c|c|}
\hline \multirow[t]{2}{*}{ SNP } & \multicolumn{2}{|l|}{ PCR primers } & \multirow{2}{*}{$\begin{array}{l}\text { TaqMan }{ }^{\circledR} \text { probes/extension } \\
\text { primers }\end{array}$} \\
\hline & Sense & Antisense & \\
\hline $\begin{array}{l}\text { TGFB1 pos. }-509 \mathrm{C} / \mathrm{T} \\
\text { (promoter) }\end{array}$ & $\begin{array}{l}\text { AAG GAG AGC AAT TCT TAC } \\
\text { AGG TGT CT }\end{array}$ & GCC TCC GGA GGG TGT CA & $\begin{array}{l}\text { VIC-CCA TCC CTC AGG TGT, } \\
\text { 6FAM-CAT CCT TCA GGT GTC }\end{array}$ \\
\hline $\begin{array}{l}\text { TGFB1 codon } 10 \text { Leu/Pro } \\
\text { (T } 869 \mathrm{C} \text { ) }\end{array}$ & GCG CTC TCG GCA GTG C & CCA GGC GTC AGC ACC AGT A & $\begin{array}{l}\text { VIC-AGC AGC GGC AGC A, } \\
\text { 6FAM-CAG CAG CAG CAG C }\end{array}$ \\
\hline $\begin{array}{l}\text { TGFB1 codon } 25 \text { Arg/Pro } \\
\text { (G } 915 \mathrm{C} \text { ) }\end{array}$ & $\begin{array}{l}\text { CTG CTA CCG CTG CTG TGG } \\
\text { CTA }\end{array}$ & TCC GCT TCA CCA GCT CCA & $(\mathrm{T})_{19} \mathrm{GGT}$ GCT GAC GCC TGG CC \\
\hline $\begin{array}{l}\text { SOD2 codon } 16 \mathrm{Val} / \mathrm{Ala} \\
\text { (T } 47 \mathrm{C} \text { ) }\end{array}$ & CTG CGT AGA CGG TCC CG & TGA TGT GAG GTT CCA GGG C & GGA GCC CAG ATA CCC CAA A \\
\hline $\begin{array}{l}\text { XRCC1 codon } 399 \mathrm{Arg} / \mathrm{Gln} \\
\text { (G } 28152 \mathrm{~A})\end{array}$ & CGC ATC GTG CGT AAG GAG & GGA CCA CCT GTG TTC TCC G & GGC GGC TGC CCT CCC \\
\hline $\begin{array}{l}\text { XRCC } 3 \text { codon } 241 \mathrm{Thr} / \mathrm{Met} \\
\text { (C } 18067 \mathrm{~T})\end{array}$ & GAT GCA CAG CAC AGG GCT C & GGT CAT CGA CTC GGT GGC & TGC TCA GCT CAC GCA GC \\
\hline $\begin{array}{l}\text { APEX codon } 148 \text { Asp/Glu } \\
\text { (T } 2107 \mathrm{G} \text { ) }\end{array}$ & $\begin{array}{l}\text { CTA TTG ATG CCT AAT GCC TGA } \\
\text { ACT C }\end{array}$ & TTC AGC CAC AAT CAC CCG & $\begin{array}{l}(\mathrm{T})_{5} \text { ATT CTG TTT CAT TTC TAT } \\
\text { AGG CGA }\end{array}$ \\
\hline ATM codon 1853 Asp/Asn & GAC TTT TGT CAG ACT GTA CTT & TCC CTG AAC ATG TGT AGA & CTT GAT TCA TGA TAT TTT ACT \\
\hline (G $5557 \mathrm{~A})$ & CCA TAC TTG & AAG CAG & CCA A \\
\hline ATM codon $1853 \mathrm{Asp} / \mathrm{Val}$ & GAC TTT TGT CAG ACT GTA CTT & TCC CTG AAC ATG TGT AGA & CAG ATT TCT CCA TGA TTC ATT \\
\hline (A $5558 \mathrm{~T}$ ) & CCA TAC TTG & AAG CAG & TGT A \\
\hline
\end{tabular}


Table 2

Putative risk alleles and distribution of pairs as to the number of risk alleles in the case compared to the matched control

\begin{tabular}{|c|c|c|c|c|c|}
\hline \multirow[t]{2}{*}{ SNP } & \multirow[t]{2}{*}{$\begin{array}{l}\text { Putative } \\
\text { risk allele }\end{array}$} & \multicolumn{3}{|c|}{$\begin{array}{l}\text { Distribution of pairs as to no. of risk alleles in the case compared to the } \\
\text { matched control }\end{array}$} & \multirow[t]{2}{*}{$\begin{array}{l}\text { Wilcoxon signed } \\
\text { ranks test } P \text {-value }\end{array}$} \\
\hline & & Case $>$ control & Case $<$ control & Case $=$ control & \\
\hline TGFB1 pos. -509 & $\mathrm{~T}$ & 11 & 3 & 12 & 0.018 \\
\hline TGFB1 codon 10 & Pro & 11 & 1 & 14 & 0.005 \\
\hline TGFB1 codon 25 & Pro & 3 & 3 & 20 & $>0.99$ \\
\hline SOD2 codon 16 & Ala & 8 & 8 & 10 & 0.82 \\
\hline XRCC1 codon 399 & Arg & 5 & 4 & 17 & 0.67 \\
\hline$X R C C 3$ codon 241 & Thr & 7 & 9 & 10 & 0.67 \\
\hline APEX codon 148 & Glu & 7 & 8 & 11 & 0.64 \\
\hline ATM codon 1853 & Gln & 7 & 4 & 15 & 0.48 \\
\hline
\end{tabular}

statistically significantly different between matched pairs of cases and controls (Table 2).

\section{Results and discussion}

The study demonstrated a significant association between the TGFB1 codon 10 Leu/Pro SNP and risk of altered breast appearance after radiotherapy. In 11 pairs, the index case had a higher number of Pro alleles than the matched control, whereas only one pair was characterized by the opposite finding $(P=0.005)$. A statistically significant association was also found for the TGFB1 position - 509 C/T SNP. In 11 pairs, the index case had a higher number of $\mathrm{T}$ alleles than the matched control whereas in only three pairs, the matched control had a higher number of $T$ alleles than the case $(P=0.018)$. For the remaining SNPs, the study did not provide any significant associations with risk of radiotherapy complications (Table 2). No patients had the ATM codon 1853 Asp/Val (A 5558 T) polymorphism.

Interestingly, both previously published studies addressing the influence of TGFB1 SNPs on clinical radiosensitivity established significant associations between the TGFB1 codon 10 Pro allele as well as the position -509 T allele and increased risk of radiation induced late complications $[2,14]$. Taking the present study into account, consistent evidence is accumulating that the risk of late normal tissue morbidity after radiotherapy is modulated by the TGFB1 SNP genotype. A genetic linkage pattern has previously been described for the TGFB1 SNPs $[3,5,17]$. Even though the functional impact of the individual SNPs remain to be elucidated, the present study seems to indicate that the correlation observed for the TGFB1 position - 509 SNP could be a result of genetic linkage with the codon 10 SNP. This assumption is supported by a recent in vitro study suggesting that the TGFB1 codon 10 SNP has a considerable impact on the TGF $\beta$-1 secretion rate [8]. For the remaining SNPs, the present study did not confirm the associations previously observed in the Danish breast cancer patient cohort $[2,15]$. This discrepancy may be attributed to several factors. Due to small sample size, the present study provided very limited statistical power to rule out a modest effect of the assessed SNPs. This problem was particularly pronounced for relatively uncommon polymorphisms such as the ATM codon 1853 and TGFB1 codon 25 SNPs. Regarding the ATM codon 1853 SNP, seven pairs were identified in which the index case had a higher number of Gln alleles than the matched control. Four pairs were characterized by the opposite finding (Table 2). Even though this skewing in the distribution was far from reaching statistical significance, it might be of interest since the codon 1853 Asn allele has previously been linked to increased risk of radiation induced normal tissue reactions $[4,15]$. In the present investigation, the primary endpoint was (grade 1 or 2 ) alteration of breast appearance assessed by serial photography $[16,18]$. In contrast, the 41 Danish patients were evaluated for (grade 3) subcutaneous induration by manual palpation [2]. From a biological point of view these endpoints are hardly equivalent. In the two studies, the length of follow-up was within the same range. However, as demonstrated in two recent papers, factors related to the kinetics of late normal tissue reactions may still hinder the detection of associations, especially for genetic alterations with only moderate impact on normal tissue complication risk [7,11].

In conclusion, the present study demonstrated significant associations between the TGFB1 codon 10 Pro allele as well as the TGFB1 position $-509 \mathrm{~T}$ allele and risk of altered breast appearance after radiotherapy. The remaining associations previously observed in the cohort of 41 Danish breast cancer patients could not be confirmed. These findings should encourage further efforts to unravel the genetic basis of clinical radiosensitivity and emphasize the need for large studies that provide sufficient statistical power to detect and quantify rather small differences in radiosensitivity.

\section{Acknowledgements}

We are grateful to Mrs Anita Ashton and Mrs Jane Hanson for assistance in the collection of skin biopsies, to Dr John Peacock for generating fibroblast strains from which DNA was isolated, to $\mathrm{Mr}$ Stephen Ebbs and Mr John Bristol for allowing us to contact their patients and to the research volunteers themselves for consenting to tissue sampling. This study was supported by the Danish Cancer Society and conducted as part of the ESTRO GENEPI project.

\footnotetext{
* Corresponding author. Christian Nicolaj Andreassen, Department of Experimental Clinical Oncology, Aarhus University Hospital, Noerrebrogade 44, DK 8000 Aarhus C, Denmark. E-mail address: nicolaj@oncology.dk
}

Received 7 October 2004; received in revised form 6 December 2004; accepted 14 December 2004; available online 14 March 2005 


\section{References}

[1] Andreassen CN, Alsner J, Overgaard J. Does variability in normal tissue reactions after radiotherapy have a genetic basis-where and how to look for it? Radiother Oncol 2002;64: 131-40.

[2] Andreassen CN, Alsner J, Overgaard M, Overgaard J. Prediction of normal tissue radiosensitivity from polymorphisms in candidate genes. Radiother Oncol 2003;69:127-35.

[3] Andreassen CN, Sorensen FB, Overgaard J, Alsner J. Optimisation and validation of methods to assess single nucleotide polymorphisms (SNPs) in archival histological material. Radiother Oncol 2004;72:351-6.

[4] Angele S, Romestaing P, Moullan N, et al. ATM haplotypes and cellular response to DNA damage: association with breast cancer risk and clinical radiosensitivity. Cancer Res 2003;63: 8717-25.

[5] Awad MR, El Gamel A, Hasleton P, Turner DM, Sinnott PJ, Hutchinson IV. Genotypic variation in the transforming growth factor-beta1 gene: association with transforming growth factor-beta1 production, fibrotic lung disease, and graft fibrosis after lung transplantation. Transplantation 1998;66: 1014-20.

[6] Baumann M, Holscher T, Begg AC. Towards genetic prediction of radiation responses: ESTRO's GENEPI project. Radiother Oncol 2003;69:121-5.

[7] Dikomey E, Borgmann K, Peacock J, Jung H. Why recent studies relating normal tissue response to individual radiosensitivity might have failed and how new studies should be performed. Int J Radiat Oncol Biol Phys 2003;56:1194-200.

[8] Dunning AM, Ellis PD, McBride S, et al. A transforming growth factorbeta1 signal peptide variant increases secretion in vitro and is associated with increased incidence of invasive breast cancer. Cancer Res 2003;63:2610-5.

[9] Green H, Ross G, Peacock J, Owen R, Yarnold J, Houlston R. Variation in the manganese superoxide dismutase gene (SOD2) is not a major cause of radiotherapy complications in breast cancer patients. Radiother Oncol 2002;63:213-6.

[10] Hall EJ, Schiff PB, Hanks GE, et al. A preliminary report: frequency of A-T heterozygotes among prostate cancer patients with severe late responses to radiation therapy. Cancer J Sci Am 1998;4:385-9.

[11] Jung H, Beck-Bornholdt HP, Svoboda V, Alberti W, Herrmann T. Quantification of late complications after radiation therapy. Radiother Oncol 2001;61:233-46.

[12] Moullan N, Cox DG, Angele S, Romestaing P, Gerard JP, Hall J. Polymorphisms in the DNA repair gene XRCC1, breast cancer risk, and response to radiotherapy. Cancer Epidemiol Biomarkers Prev 2003;12:1168-74.

[13] Peacock J, Ashton A, Bliss J, et al. Cellular radiosensitivity and complication risk after curative radiotherapy. Radiother Oncol 2000;55:173-8.

[14] Quarmby S, Fakhoury H, Levine E, et al. Association of transforming growth factor beta-1 single nucleotide polymorphisms with radiation-induced damage to normal tissues in breast cancer patients. Int J Radiat Biol 2003;79:137-43.

[15] Rosenstein BS, Andreassen CN, Alsner J, et al. Possible correlation between the ATM codon 1853 ASP/ASN polymorphism and risk of radiation-induced fibrosis (meetings abstract ESTRO 23). Radiother Oncol 2004;73:24.

[16] Shayeghi M, Seal S, Regan J, et al. Heterozygosity for mutations in the ataxia telangiectasia gene is not a major cause of radiotherapy complications in breast cancer patients. $\mathrm{Br}$ J Cancer 1998;78:922-7.

[17] Syrris P, Carter ND, Metcalfe JC, et al. Transforming growth factor-beta1 gene polymorphisms and coronary artery disease. Clin Sci (London) 1998;95:659-67.

[18] Yarnold J, Ashton A, Bliss J, et al. Fractionation sensitivity and dose response of late adverse effects in the breast after radiotherapy for early breast cancer: long-term results of a randomised trial. Radiother Oncol 2005;75:9-17. 